\title{
The R2R3-MYB transcription factor PaMYB10 is involved in anthocyanin biosynthesis in apricots and determines red blushed skin
}

Wanpeng $\mathrm{Xi}^{1,2}$, Jing Feng ${ }^{2}$, Yu Liu' ${ }^{2}$, Shikui Zhang ${ }^{3}$ and Guohua Zhao ${ }^{1 *}$

\begin{abstract}
Background: The majority of apricot (Prunus armeniaca L.) cultivars display orange or yellow background skin, whereas some cultivars are particularly preferred by consumers because of their red blushed skin on the background.

Results: In this study, two blushed ('Jianali' and 'Hongyu') and two nonblushed ('Baixing' and 'Luntaixiaobaixing') cultivars were used to investigate the formation mechanism of blushed skin in apricots. High-performance liquid chromatography (HPLC) analysis showed that the blushed cultivars accumulated higher cyanidin-3-O-glucoside, cyanidin-3-O-rutinoside and peonidin-3-O-rutinoside levels during fruit ripening than the nonblushed cultivars. Based on coexpression network analysis (WGCNA), a putative anthocyanin-related R2R3-MYB, PaMYB10, and seven structural genes were identified from transcriptome data. The phylogenetic analysis indicated that PaMYB10 clustered in the anthocyanin-related MYB clade. Sequence alignments revealed that PaMYB10 contained a bHLH-interaction motif ([DE]LX2[RK]×3Lx6LX3R) and an ANDV motif. Subcellular localization analysis showed that PaMYB10 was a nuclear protein. Real-time qRT-PCR analysis demonstrated that the transcript levels of PaMYB10 and seven genes responsible for anthocyanin synthesis were significantly higher in blushed than in nonblushed apricots, which was consistent with the accumulation of anthocyanin. In addition, bagging significantly inhibited the transcript levels of PaMYB10 and the structural genes in 'Jianali' and blocked the red coloration and anthocyanin accumulation. Transient PaMYB10 overexpression in 'Luntaixiaobaixing' fruits resulted in the red blushed skin at the maturation stage.
\end{abstract}

Conclusions: Taken together, these data reveal that three anthocyanins are responsible for the blushed skin of apricots, identify PaMYB10 as a positive regulator of anthocyanin biosynthesis in apricots, and demonstrate that blush formation depends on light.

Keywords: Prunus armeniaca, Anthocyanin, MYB transcription factor, WGCNA, Cloning, GRT-PCR

\footnotetext{
* Correspondence: zhaogh@swu.edu.cn

${ }^{1}$ College of Food Science, Southwest University, Chongqing 400715, China

Full list of author information is available at the end of the article
}

(c) The Author(s). 2019 Open Access This article is distributed under the terms of the Creative Commons Attribution 4.0 International License (http://creativecommons.org/licenses/by/4.0/), which permits unrestricted use, distribution, and reproduction in any medium, provided you give appropriate credit to the original author(s) and the source, provide a link to the Creative Commons license, and indicate if changes were made. The Creative Commons Public Domain Dedication waiver (http://creativecommons.org/publicdomain/zero/1.0/) applies to the data made available in this article, unless otherwise stated. 


\section{Background}

Apricot (Prunus armeniaca L.) is a widely cultivated temperate fruit tree species [1] with fruit that contains many types of carotenoids and presents with orange or yellow skin. Xinjiang is one of the primary centers of apricot domestication worldwide and is also the main cultivation area in China; almost 200 varieties are cultivated in this region [2]. Among these varieties, some cultivars with a red blush on the orange or yellow background skin are particularly preferred by consumers due to their beautiful color and high nutritional value $[3,4]$. However, the mechanism underlying blush formation in these cultivars is unknown.

Anthocyanins are water-soluble flavonoid pigments that accumulate in the vacuoles of many flowers, fruits, seeds, and vegetables, where they contribute to the red, purple and blue coloration [5]. As the primary color determinant and antioxidant components, the accumulation of anthocyanin pigments in fruit is an important indicator of health-promoting property $[6,7]$. Therefore, understanding the regulation of anthocyanin biosynthesis in fruits is important for development of anthocyanin-rich foods for our diet.

Anthocyanins are synthesized from a branch of the flavonoid pathway, which has been extensively studied in many plants [8-10]. In general, anthocyanin biosynthesis at the transcriptional level is controlled by a complex of DNA-binding R2R3 MYB transcription factors, MYC-like basic helix-loop-helix (bHLH) proteins and WD40 proteins [11-13]. MYB members of this complex can separately play the regulatory role in anthocyanin biosynthesis [14, 15], and have been identified in various fruit crops, including grapes [16], apples [17], pears [18] and peaches [19].

MYB TFs have been reported to have diverse functions in controlling plant pathways, such as secondary metabolism, development, signal transduction, and disease resistance [20]. These TFs are characterized by a structurally conserved DNA-binding domain consisting of single or multiple imperfect repeats. Among these MYB TFs, the two repeat class (R2R3) is the largest, with 133 R2R3 MYB TFs reported in Arabidopsis [21, 22] and 108 reported in grapes [23]. It has been clearly elucidated that R2R3 MYB TFs are the key to determine the spatial and temporal patterning of anthocyanin production in most plant species [7, 15, 24]. Hence, the isolation and characterization of MYB TFs associated with anthocyanin biosynthesis is an important step towards understanding and manipulating fruit coloration [25].

Recently, great progress has been made in elucidating the molecular control of the anthocyanin pathway in many horticulture crops [14]. Anthocyanin biosynthesis is enhanced by sunlight in apples, pears, and peaches
[26]. In apricots, although blushed skin is observed in some cultivars, no information about the mechanism of its development is available at present. In the present study, we determined the main pigments contributing to fruit blushed skin and identified one R2R3 MYB transcription factor and seven structural genes of the anthocyanin biosynthetic pathway based on coexpression network analysis (WGCNA). The potential roles of MYB in anthocyanin accumulation in apricots were investigated by bagging and transient overexpression. Our results suggest that PaMYB10 is responsible for controlling anthocyanin biosynthesis and is involved in the development of blushed skin in apricots and that the process is regulated by light.

\section{Results}

Changes in basic quality parameters during fruit development and ripening

From the fruitlet (F) to fully ripe (FR) stages, the fruit weight of all experimental cultivars increased rapidly and presented a typical S-shaped curve (Fig. 1a), whereas the fruit firmness declined sharply throughout the development period (Fig. 1b). The TSS concentration in all fruits increased remarkably with fruit development (Fig. 1c) and the TA concentration decreased significantly (Fig. 1d), which showed that the five developmental stages completely covered the full process of fruit development and ripening. The four apricot cultivars showed a gradual color change from the turning $(\mathrm{T})$ stage. The $\mathrm{a}^{*}$ value increased during ripening and was positive at the enlargement $(\mathrm{E})$, commercial maturation (CM) and FR stages in 'HY' and 'JNL', indicating that the fruit was turning red, whereas the value was negative during the whole ripening process in 'LT' and 'BX' (Additional file 1: Table S1). At the CM stage, a blush appeared on the fruit skin of 'JNL' and 'HY', whereas the skins of 'LT' and 'BX' did not display blush (Fig. 2a). Generally, the color index for red grape (CIRG) is used to evaluate the redness of fruit. The CIRG values of all fruit peels increased with development, although the CIRG values of the 'HY' and 'JNL' fruit peels were significantly higher than those of 'BX' and 'LT' (Fig. 2b).

\section{Changes in the anthocyanin content during fruit development and ripening}

Three anthocyanins (cyanidin-3-O-glucoside, cyanidin-3O-rutinoside and peonidin-3-O-rutinoside) were identified from the apricot peels (Fig. 3). During development and ripening, the anthocyanin contents of 'BX' and ' $\mathrm{LT}$ ' remained very low, whereas those of 'JNL' and 'HY' increased significantly. For ripe fruit, the total anthocyanin concentrations in 'JNL' and 'HY' were $35.05 \mathrm{mg} / \mathrm{kg}$. FW and $39.17 \mathrm{mg} / \mathrm{kg} . \mathrm{FW}$, respectively, which were significantly higher than those in 'BX' $(0.09 \mathrm{mg} / \mathrm{kg} \cdot \mathrm{FW})$ and 'LT' $(0.07 \mathrm{mg} / \mathrm{kg}$.FW). Interestingly, we detected cyanidin-3-O- 


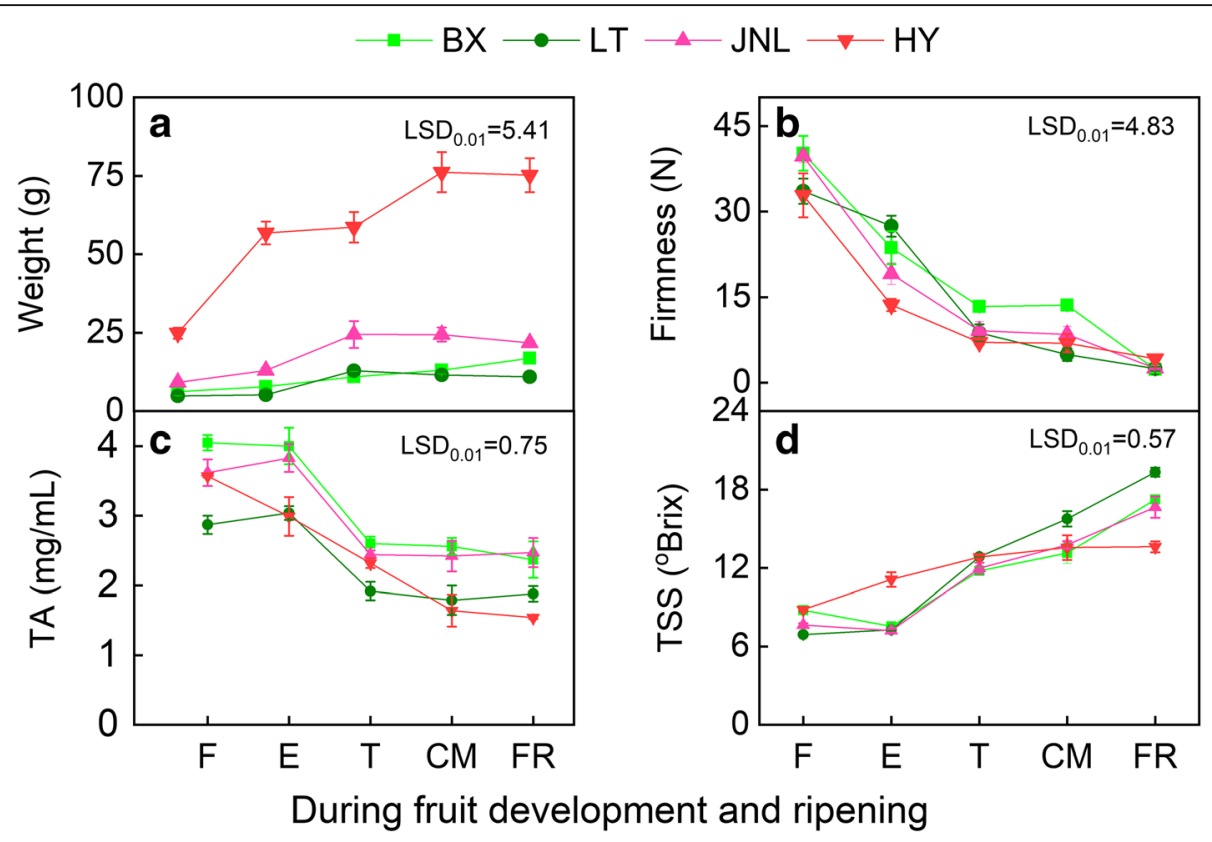

Fig. 1 Changes in basic quality indexes during fruit development and ripening. a Fruit weight. b fruit firmness. c TSS, total soluble solid. d TA, titratable acid. Error bars are \pm SE of the means of three biological replicates. The least significant difference (LSD) test at the $1 \%$ level was performed for all pairwise comparisons

rutinoside in the red cultivars; it accounted for $58.8 \%$ and $62.7 \%$ of the total anthocyanins in 'JNL' and 'HY', respectively, but was not detected in the 'BX' and 'LT' fruit peels.

\section{Screening of candidate genes by WGCNA}

We performed a WGCNA to investigate the transcription factors and structural genes responsible for anthocyanin biosynthesis. We found that the blue and green modules were significantly positively correlated with cyanidin-3-O-glucoside, with correlation coefficients of $0.92\left(\mathrm{e}\right.$-value $\left.=4 \times 10^{-4}\right)$ and $0.94\left(\mathrm{e}\right.$-value $\left.=1 \times 10^{-4}\right)$, respectively. Similar significant correlations were found between the two modules and cyanidin-3-O-rutinoside and peonidin-3-O-rutinoside (Fig. 4). Based on the correlation coefficients between genes in the blue and green modules and the anthocyanin compounds, and changes in Fragments Per Kilobase of transcript per Million fragments mapped (FPKM) values of these genes during fruit ripening (Fig. 5), one U1373 gene encoding MYB was located in the blue module, and seven structural genes were identified as putative candidate genes for anthocyanin biosynthesis in apricots, including one chalcone isomerase (CHI: U21320) and one UDP-glucose: flavonoid 3-O-glucosyltransferase (UF GT: U3633) located in the blue module and one phenylalanine ammonia lyase (PAL: U15872), one chalcone synthase (CHS: CL2328.1), one flavonoid 3hydroxylase (F3H: CL472.1), one dihydroflavonol 4- reductase (DFR: U22536), and one leucoanthocyanidin dioxygenase (LDOX: U21017) located in the green module (Additional file 2: Table S2).

\section{Phylogenetic and conserved motif analyses and subcellular localization of PaMYB10}

A bootstrapped circular phylogenetic tree was constructed based on 148 MYB genes from the apricot transcriptome and MYB transcription factors in other species. The tree showed that the 148 MYB TFs were distributed into five clades (Additional file 3: Figure S1), including flavonoid-related MYB, water stress-related MYB, phenol-related MYB, cell wall composition-related MYB, and some unknown function MYB clades. U1373 was clearly clustered with other MYB transcription factors involved in flavonoid synthesis. To further verify the results, another phylogenetic tree was generated based on U1373 and 18 flavonoid-related MYB transcription factors from other species. The second phylogenetic tree showed that U1373 belonged to the anthocyanin-related MYB clade and clearly clustered with PpMYB10, showing high homology with PbMYB10 in pear (Pyrus bretschneideri) fruit and MdMYB1 and MdMYB10 in apples (Malus domestica) (Fig. 6a) [27, 28]. Therefore, U1373 was designated PaMYB10.

Furthermore, the full-length U1373 was isolated from the peel of 'JNL' using RACE. The sequence was $675 \mathrm{bp}$ in length and contained a complete open reading frame 
a

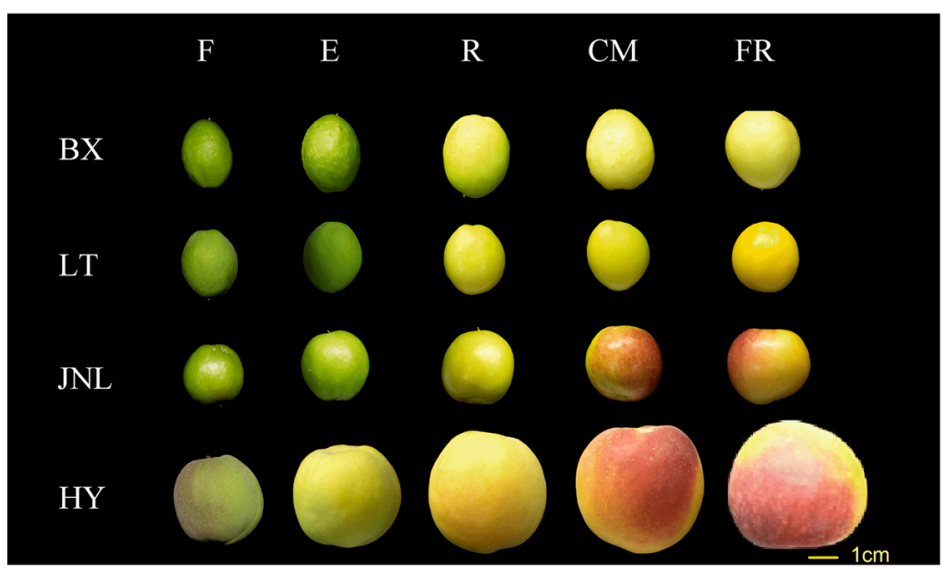

b

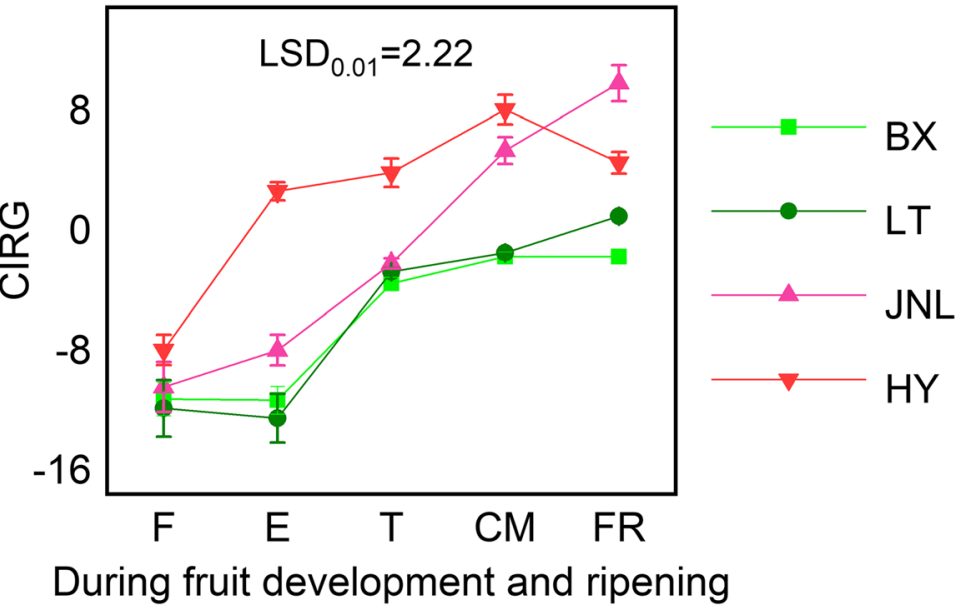

Fig. 2 Changes in fruit color during development and ripening. a Color change of apricot fruit. b CIRG, color index for red grapes. Error bars are the \pm SE of the means of three biological replicates. The least significant difference (LSD) test at the $1 \%$ level was performed for all pairwise comparisons

encoding 225 amino acids. Subsequently, comparison of the deduced amino acid sequence of PaMYB10 with those of several anthocyanin-related MYB transcription factors from other plants revealed a high degree of sequence similarity in the R2 and R3 DNA-binding domains of these proteins. As shown in Fig. 6b, a conserved KPRPR [S/T] F motif was also present in the R2R3 domain of the PaMYB10 C-terminus. Further analysis showed that the R3 DNA-binding domain contained an R/B-like bHLH interaction motif ([DE]Lx 2[RK]x3Lx6Lx3R) and an ANDV motif.

To determine the subcellular localization of PaMYB10, tobacco (Nicotiana tabacum) leaves bombarded with the recombinant vector $\mathrm{pC} 1301-\mathrm{GFP}-\mathrm{MYB10}$ and control vector pC1301-GFP were observed under a confocal laser scanning microscope. We found that the tobacco leaves with the control vector pC1301-GFP exhibited fluorescence throughout the entire cell, whereas those with the recombinant vector p163-GFP-MYB10 displayed strong fluorescence in the nucleus. These results demonstrated that the expression site of this gene was in the nucleus (Fig. 6c).
Transcript levels of PaMYB10 and structural genes related to anthocyanin synthesis

The results showed that the transcript levels of all genes in the peels of the blushed cultivars ('JNL' and 'HY') were significantly higher than those in the peels of the nonblushed cultivars ('BX' and 'LT') $(P<0.05)$ (Fig. 7). The transcript levels of PaMYB10, PaPAL, PaCHS, PaCHI, PaF3H, PaDFR, PaLDOX and PaUFGT were low in 'BX' and 'LT', with only slight upregulation observed in the $\mathrm{F}$ and $\mathrm{T}$ stages. However, the expression levels of PaMYB10 and the seven structural genes in 'JNL' and 'HY' all increased remarkably during the whole developmental period, whereas the expression patterns of PaMYB10 and most of the structural genes in 'HY' showed a double-peak pattern and reached their highest values in the $\mathrm{CM}$ stage.

The effect of bagging on gene expression and anthocyanin accumulation in the 'JNL' fruit The $\mathrm{a}^{*}$ value in bagged fruits (5.87) was significantly lower than that in nonbagged fruits (20.84) (Additional file 1: Table S1), and the CIRG values also decreased from 1.20 to 


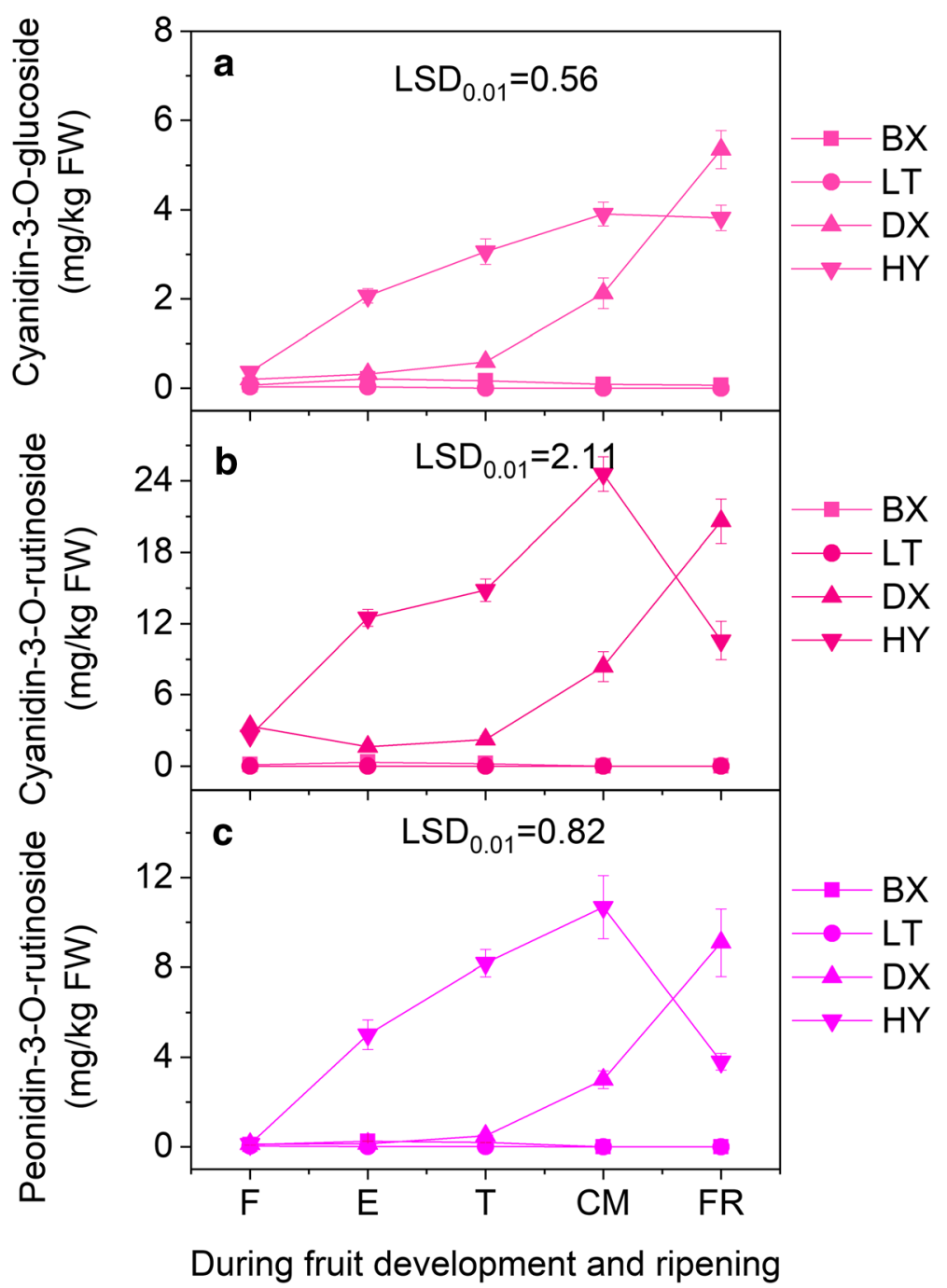

Fig. 3 Changes of anthocyanidins in apricot fruit during development and ripening. Error bars are the \pm SE of the means of three biological replicates. The least significant difference (LSD) test at the $1 \%$ level was performed for all pairwise comparisons

0.90. No blush was observed in the bagged 'JNL' fruit skin (Fig. 8a). The cyanidin-3-O-glucoside, cyanidin-3-O-rutinoside and peonidin-3-O-rutinoside contents in bagged fruits were extremely reduced from $35.05 \mathrm{mg} / \mathrm{kg} \mathrm{FW}$ to $2.17 \mathrm{mg} /$ $\mathrm{kg}$ FW in the 'JNL' peel (Fig. 8b), and the PaMYB10, PaPAL, PaCHS, PaCHI, PaF3H, PaDFR, PaLDOX and PaUFGT expression levels were also significantly lower than those in the nonbagged fruits $(P<0.05)$ (Fig. 8c).

Transient PaMYB10 overexpression increases anthocyanin production in the ' $\mathrm{LT}^{\top}$ ' fruit peel

PaMYB10 was transiently overexpressed in the 'LT' fruit peel at the CM stage. The fruit peel presented with a red blush, whereas the control fruit skin did not present with a blush (Fig. 9a). Compared with those of the control, the cyanidin-3-O-glucoside, cyanidin-3-
O-rutinoside and peonidin-3-O-rutinoside contents were significantly increased by 2.2-fold, 3-fold and 2.4-fold, respectively, in the PaMYB10-overexpressed fruits (Fig. 9b).

\section{Discussion}

Apricots with a blush on orange or yellow skin are becoming more and more popular in the market due to their colorful appearance and excellent nutritional value [29]; however, the mechanism underlying the development of these cultivars has remained elusive. In this study, we compared the anthocyanin composition and content among apricot cultivars with and without a blush. Based on the WGCNA, gene expression and gene structural analyses and transgenic experiments, we identified the R2R3MYB TF PaMYB10 as a master regulator that controlled 


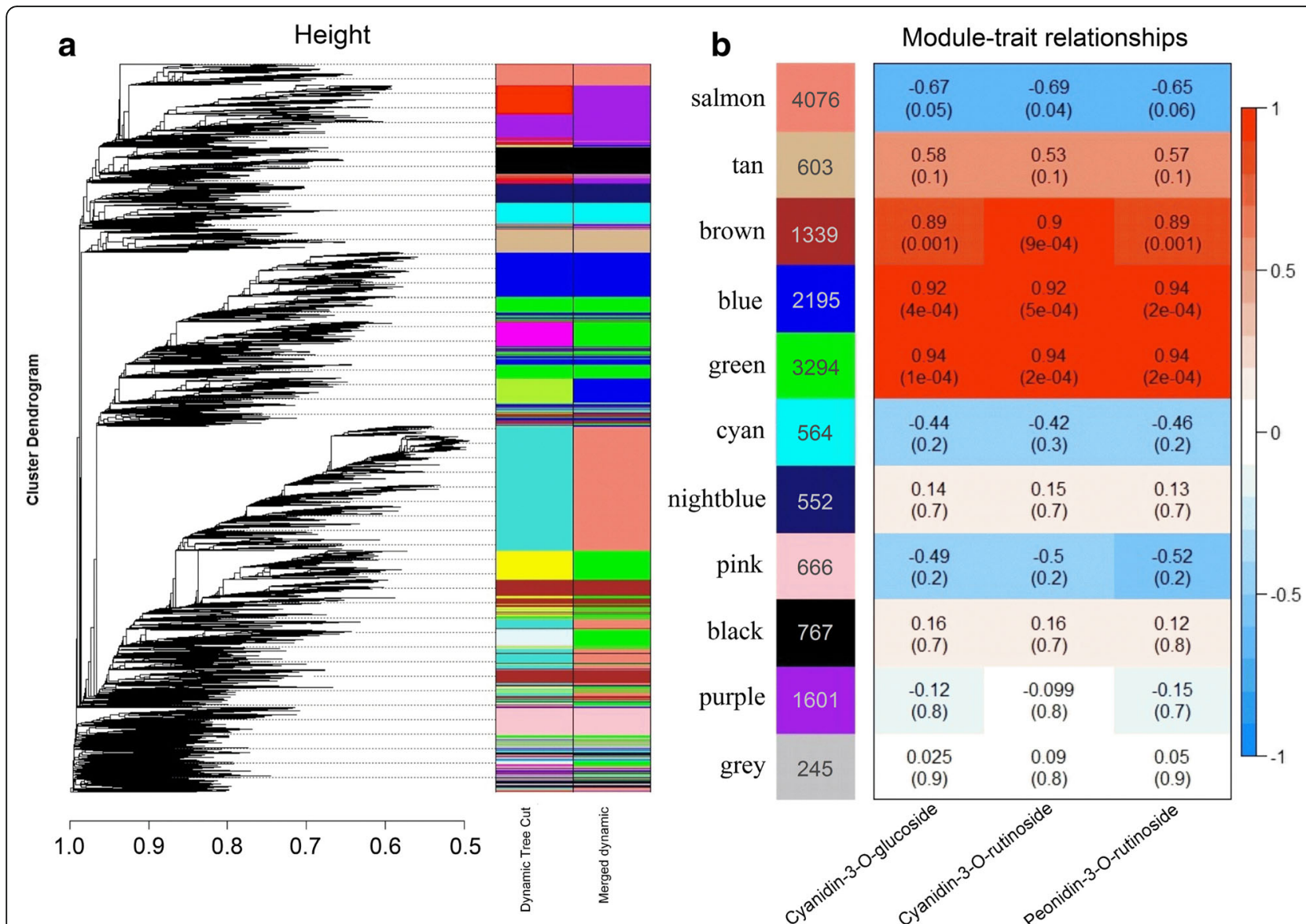

Fig. 4 Weighted gene coexpression network analysis of apricot transcriptomes during fruit ripening. a Hierarchical cluster tree showing 11 coexpressed gene modules. Each leaf in the tree represents one gene. b Module-anthocyanin correlations and corresponding $p$-values. The left panel shows 11 modules and the numbers of their member genes. The right panel is a color scale for module trait correlation from - 1 to 1

the entire anthocyanin biosynthesis process during apricot fruit ripening.

Our chemical analysis demonstrated that the total anthocyanin content in apricots with red blushed skin as well as the individual cyanidin-3-O-glucoside, cyanidin-3O-rutinoside and peonidin-3-O-rutinoside contents were significantly higher than those found in apricots without a blush (Fig. 3). In particular, remarkably high cyanidin-3O-rutinoside levels were observed in red blushed cultivars during fruit ripening but were undetectable throughout the whole ripening process in cultivars without a blush. These results showed that high levels of the three anthocyanins determined the blush coloration of the apricot fruit skin and that cyanidin-3-O-rutinoside played the dominant role in blush formation. As shown in Fig. 2a, the 'JNL' and 'HY' fruit skins turned red after the T stage, which showed that the blush depended on fruit ripening. Thus, to understand the molecular mechanism of blush formation, the T, CM and FR stages of 'JNL' fruits, which represented the full ripening process, were used for transcriptome sequencing. Using WGCNA, we identified one MYB gene and seven structural genes related to anthocyanin biosynthesis in the blue and green modules that were significantly positively correlated with cyanidin3-O-glucoside, cyanidin-3-O-rutinoside and peonidin-3O-rutinoside (Fig. 4). MYB was considered the hub gene in the anthocyanin metabolic network, which suggested a direct connection between MYB and anthocyanin biosynthesis in apricot fruit.

For functional studies, full-length PaMYB10 cDNA was cloned from 'JNL'. In the subsequent phylogenetic analysis, PaMYB10 was found to cluster with $P p M Y B 10$, PbMYB10, MdMYB1 and MdMYB10 (Fig. 6a), which are known to be positively associated with anthocyanin production [27, 28, 30, 31]. Sequence alignment found that the apricot PaMYB10 contained the typical R2 and R3 structural domains similar to other MYB members in the same cluster (Fig. 6a). Sequence analysis also showed that PaMYB10 contained an ANDV motif in the R3 domain, which is an identifier for anthocyanin-related MYBs [27]. 


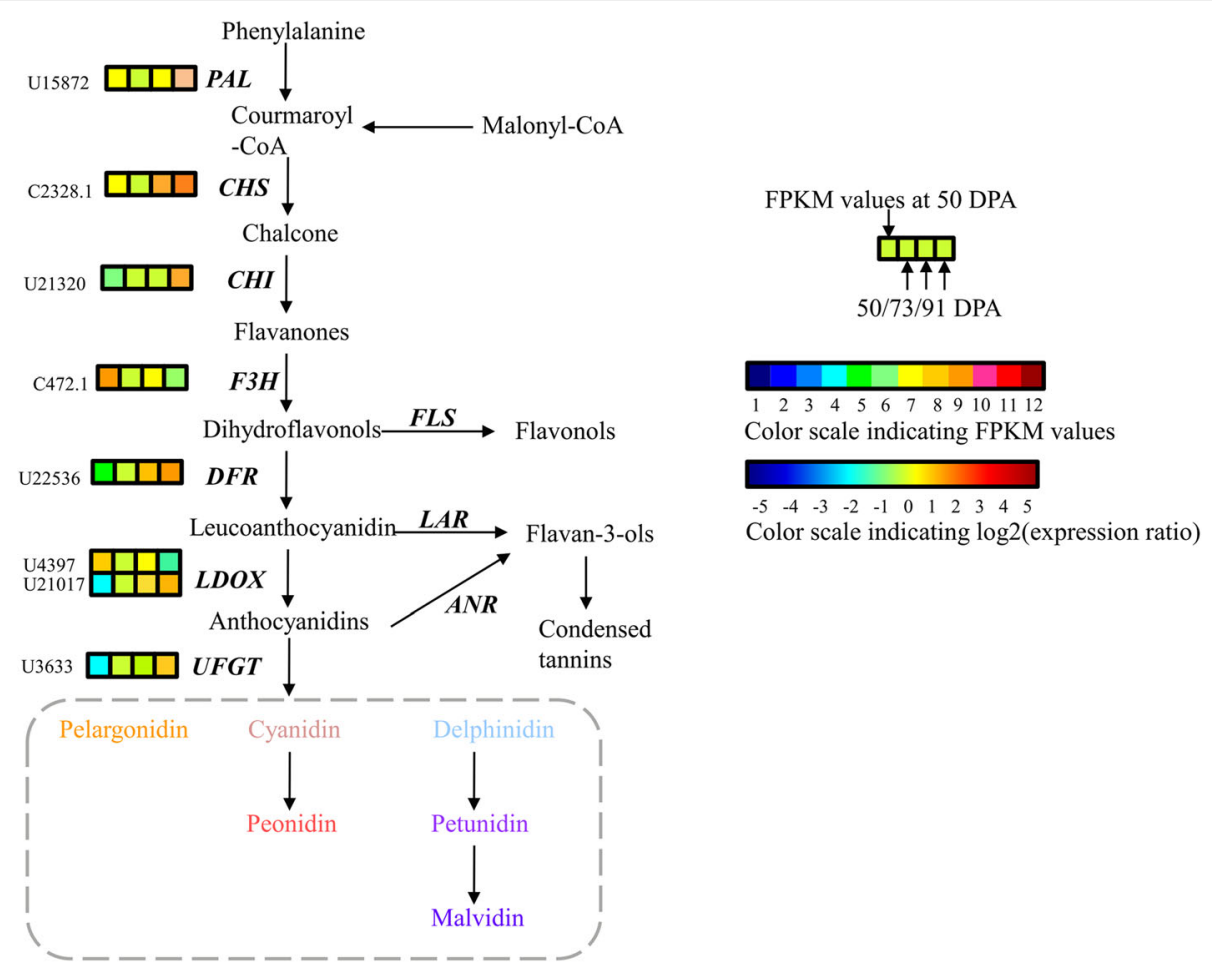

Fig. 5 Anthocyanin metabolism scheme in apricot fruit during ripening. The expression patterns of each Unigene are shown as 4 grids, with the left grid representing the FPKM value at 50 DPA, and the second to fourth grids from the left to the right representing the relative log2 (expression ratio) at 50 DPA, 73 DPA, and 91 DPA, respectively. The grids with 12 different colors from blue to wine show the absolute expression magnitude at 50 DPA, with FPKM values of $0-1,1-2,2-4,4-8,8-16,16-32,32-64,64-128,128-256,256-512,512-1024$ and over 1024 represented by colors 1 to 12 , respectively

In addition to the R2R3 domain, the KPRPR [S/T] F motif, which is a R2R3-MYB specific structure [22], was found in $P a M Y B 10$. Finally, the subcellular localization analysis revealed that PaMYB10-GFP was distributed in the nucleus (Fig. 6c). These results suggested that PaMYB10 may play an important role in regulating anthocyanin synthesis in apricot fruit.

We also compared the expression levels of PaMYB10 and structural genes related to anthocyanin biosynthesis between blushed and nonblushed apricots and found that the transcript levels of all genes in the apricots with blush were significantly higher than those in the apricots without blush during the fruit ripening process. These findings were consistent with the accumulation of anthocyanins, which proved that these genes were related to anthocyanin biosynthesis (Fig. 7). At the same time, we demonstrated that bagging resulted in a significant decrease in the accumulation of anthocyanins in 'JNL' and prevented blush formation in the fruit skin (Fig. 8b), which coincided with a dramatic decrease in the transcript levels of PaMYB10 and the structural genes (Fig. 8c). These results further confirmed that PaMYB10 was a key gene involved in the control of anthocyanin biosynthesis and simultaneously suggested that light could affect blush formation on apricots. These findings are in agreement with previous reports in other fruits linking the light-mediated mechanism involved in the regulation of anthocyanin biosynthesis with ripening-related anthocyanin accumulation [14].

In plants, transcriptional regulation of structural genes by transcription factors appears to be the major mechanism controlling anthocyanin biosynthesis [14, 32, 33]. Most MYBs involved in the control of anthocyanin biosynthesis are positive regulators that enhance the expression of structural anthocyanin pathway genes. However, repressors have also been characterized, such as $V v M Y B 4$ in the berries of grapevines and FaMYB1 in strawberries (Fragaria $x$ ananassa Duch.) [23, 34]. In the present study, transient overexpression of a single PaMYB10 gene in 'LT' apricots promoted blush formation and significantly increased the transcript abundance of PaMYB10 and structural genes responsible for anthocyanin biosynthesis. These results suggested a central positive role for PaMYB10 in regulation of anthocyanin synthesis.

R2R3 MYB TF regulation can occur at different steps of the anthocyanin biosynthesis pathway. For example, R2R3 MYB TFs in perilla (Perilla frutescens) 
a

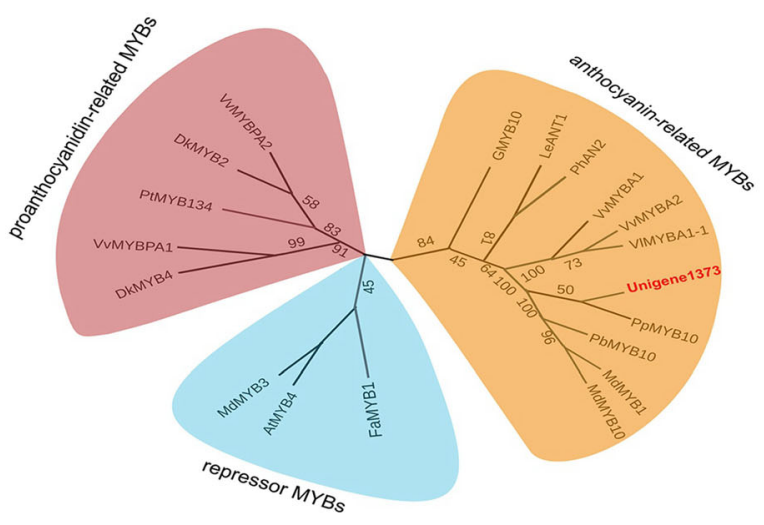

b

R2
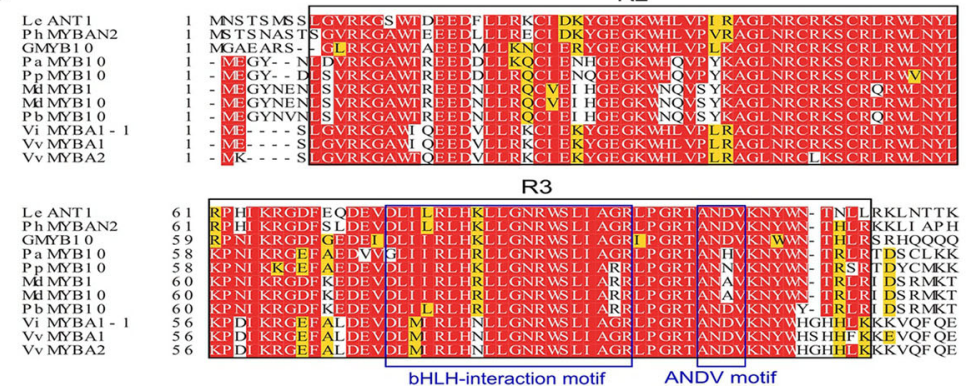

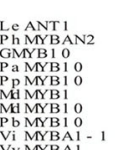

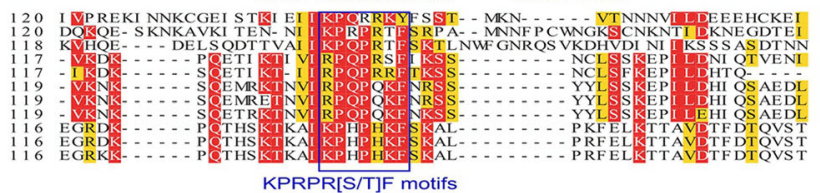

Vi MYBA1
VvMYBA1
Vv MYBA2

KPRPR[S/T]F motifs

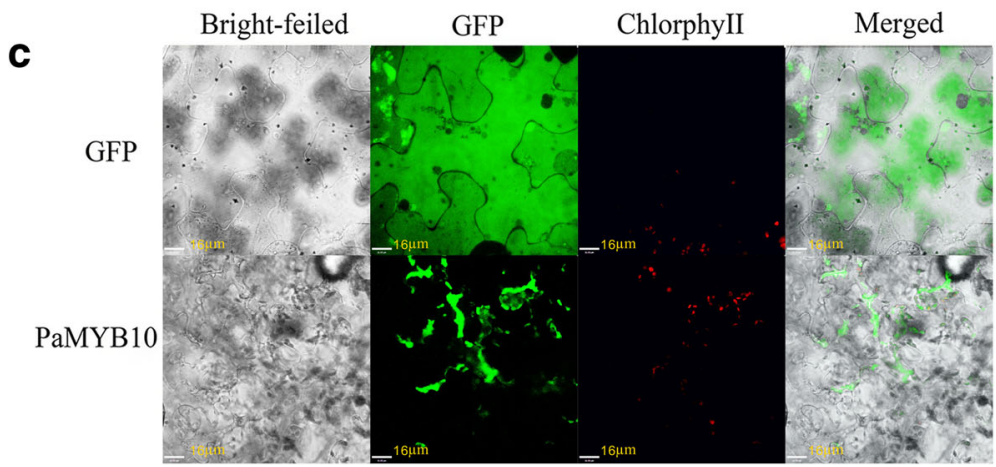

Fig. 6 Sequence analysis and subcellular localization of PaMYB10. a Phylogenetic tree of the U1373 gene and other MYB transcription factors from different plants. b Amino acid sequence alignment of PaMYB10 with other species. The red font indicates 100\% homology, the black boxes indicate the R2R3 domain, and the blue boxes indicate the bHLH-interaction domain, ANDV, and KPRPR [S/T] F motifs. c Subcellular localization of PaMYB10 in Nicotiana benthamiana leaves. The enhanced green fluorescent protein (GFP)-fused PaMYB10 was transiently expressed in N. benthamiana leaves and imaged with a confocal fluorescence microscope

control transcription of all structural genes involved in anthocyanin biosynthesis [35]. Overexpression of AtPAP1 and AtPAP2 strongly induce anthocyanin accumulation in Arabidopsis as a result of upregulation of all genes in the anthocyanin biosynthetic pathway [36, 37]. Similarly, our results showed that almost all genes responsible for anthocyanin biosynthesis were up-regulated in overexpressed apricot fruit of PaMYB10, which suggested the global regulation role of PaMYB10 for the whole biosynthesis pathway of anthocyanin biosynthesis in apricot, but future work is still required to investigate which genes are targeted by the MYB TF. 


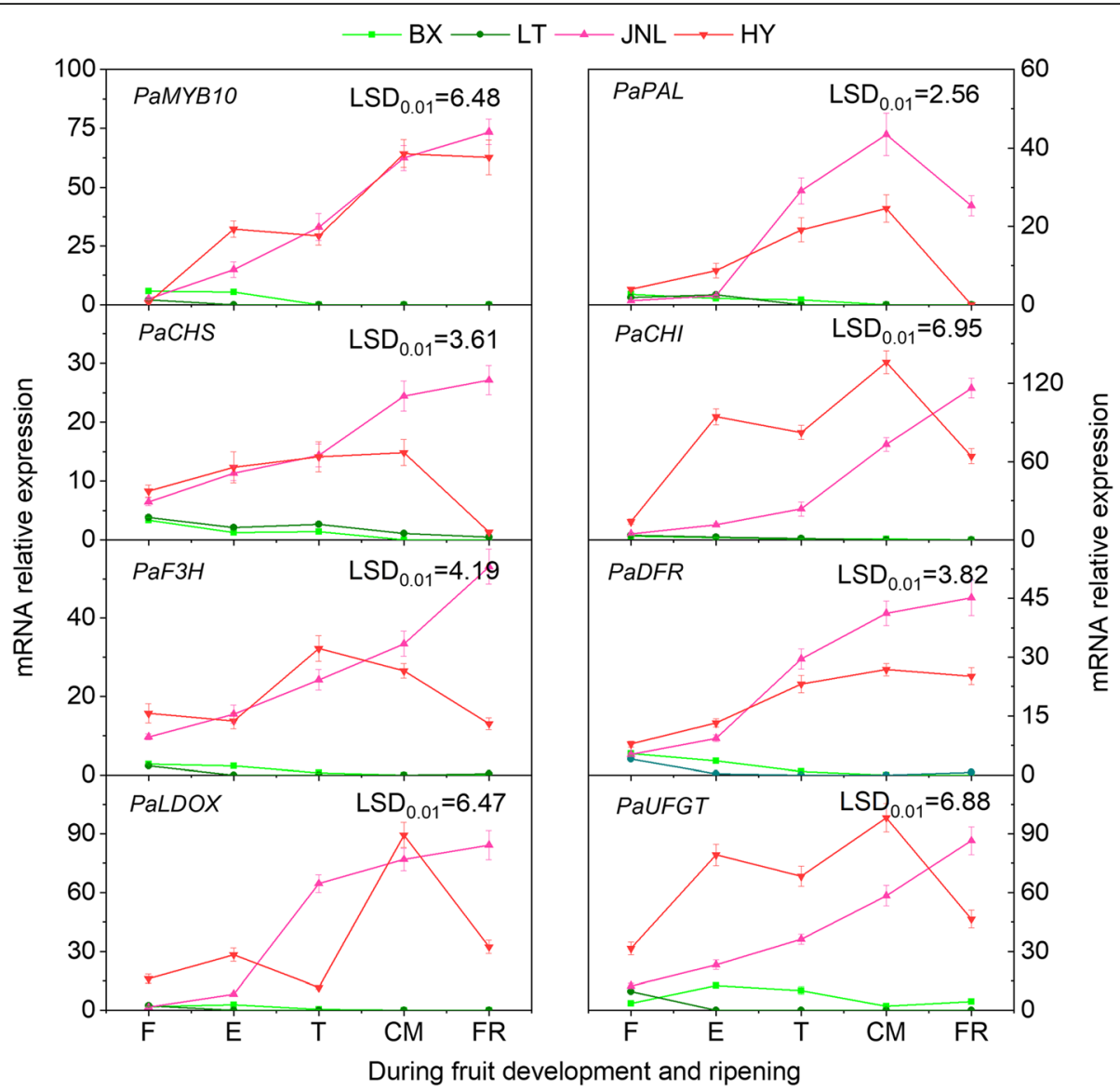

Fig. 7 The transcript levels of genes involved in anthocyanin biosynthesis in fruit peels. Error bars are the \pm SE of the means of three biological replicates. The least significant difference (LSD) test at the $1 \%$ level was performed for all pairwise comparisons

In general, regulation of the anthocyanin biosynthesis pathway is controlled by the MYB-bHLHWD40 (MBW) complex [7, 23]. But some anthocyanin biosynthesis-related MYB transcription factors that are activated without a bHLH partner have been identified in grapevines and Arabidopsis thaliana [24, 38]. In the present study, the amino acid signature ([DE]Lx2[RK]x3Lx6Lx3R) of the R3 domain, which was used to connect the MYB and bHLH proteins [21], was found in the PaMYB10 protein, suggesting a potential interaction between the bHLH protein and PaMYB (Fig. 6b), so more evidence should be provided for whether other TFs may be coordinately involved in the process.

\section{Conclusions}

In the study, we found that the red blushed skin of apricots is attributed to the accumulations of cyanidin-3-Oglucoside, cyanidin-3-O-rutinoside and peonidin-3-Orutinoside during fruit ripening. The R2R3 MYB TF
PaMYB10 was identified as a central gene related to the regulation of anthocyanin biosynthesis and blush formation, and the process is regulated by light.

\section{Methods}

\section{Plant materials}

Four varieties of apricot ['Jianali' (JNL) and 'Hongyu' (HY) (fruit with blushed skin) and 'Baixing' (BX) and 'Luntaixiaobaixing' (LT) (fruit without blushed skin)] cultivated at the National Fruit Tree Germplasm Repository, Academy of Xinjiang Agricultural Sciences, Luntai, Xingjiang, China, were used as the experimental materials. Every six individual trees were treated as one replication, and fruits were harvested at the fruitlet (F, $30 \mathrm{DAB})$, enlargement (E, $45 \mathrm{DAB}$ for 'BX, 'LT, 'JNL' and 'HY'), turning ( $\mathrm{T}, 60 \mathrm{DAB}$ for 'BX' and 'LT' and $54 \mathrm{DAB}$ for 'JNL' and 'HY'), commercial maturation (CM, 75 DAB for 'BX' and 'LT' and 72 $\mathrm{DAB}$ for ' $\mathrm{NL}$ ' and 'HY') and fully ripe (FR, $90 \mathrm{DAB}$ for 'BX' and 'LT' and 82 DAB for 'JNL' and 'HY') stages. In addition, fifty 'JNL' fruits were bagged before the T stage 

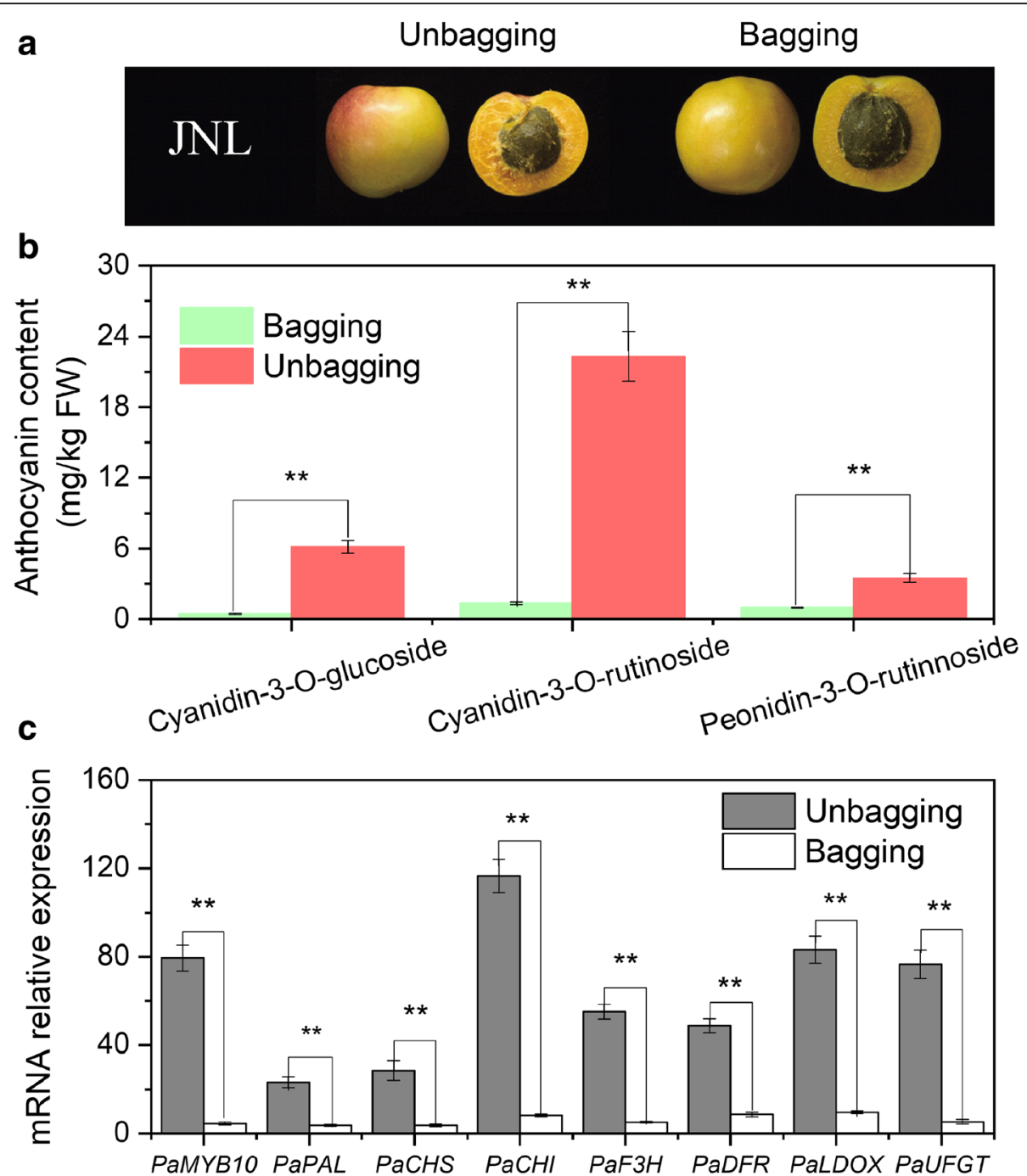

Fig. 8 Effect of bagging on the anthocyanin content and the transcript levels of genes related to anthocyanin biosynthesis in 'JNL'. a Fruit color. b Changes in the anthocyanin content. c Transcripts levels of genes related to anthocyanin biosynthesis. Error bars are the \pm SE of the means of three biological replicates. The double asterisk indicates statistically significant differences by Duncan's test at the 1\% level

and harvested at the FR stage. Twenty of the fifty harvested fruits were used to measure basic physiological indexes, such as the fruit size, chromatic aberration, total soluble solids (TSS), and titratable acidity (TA). Three biological replicates for each sample were used. The peel and pulp were separated from the remaining 30 sampled fruits, immediately frozen in liquid nitrogen, and stored at $80^{\circ} \mathrm{C}$ prior to use.

\section{Anthocyanin extraction and identification}

Total anthocyanins were extracted from the fruit peels according to the methods described by our previous study with slight modifications [39]. Frozen peels (1 g) were ground to a fine powder by an IKA A11 analytical grinder (IKA, Staufen, Baden-Württemberg, Germany) and extracted using $3 \mathrm{~mL}$ of $2 \%$ formic acid for $24 \mathrm{~h}$ at $4{ }^{\circ} \mathrm{C}$ in the dark. The extraction was evaporated using a rotary evaporator for the next step. The leachate residue was dissolved in methanolic hydrochloric acid solution $(\mathrm{pH}=2.3)$ and then adjusted to a final volume of $1.5 \mathrm{~mL}$. Subsequently, the clear supernatant was collected and filtered to remove cell debris. Anthocyanins in the samples were analyzed using a Waters HPLC system with a diode array detector (Waters, Milford, MA, USA) at $40^{\circ} \mathrm{C}$. Each sample $(20 \mu \mathrm{L})$ was loaded onto a reversedphase $\mathrm{C}_{18}$ analytical column $(4.6 \mathrm{~mm}$ i.d. $\times 250 \mathrm{~mm}$ i.d., $5 \mu \mathrm{m})($ Nacalai Tesque, Kyoto, Japan) and eluted using a mobile phase consisting of solvent A ( $2 \%$ formic acid in water) and solvent B ( $2 \%$ formic acid and $98 \%$ acetonitrile) for $40 \mathrm{~min}$ at a flow rate of $1 \mathrm{~mL}$ per min. The 


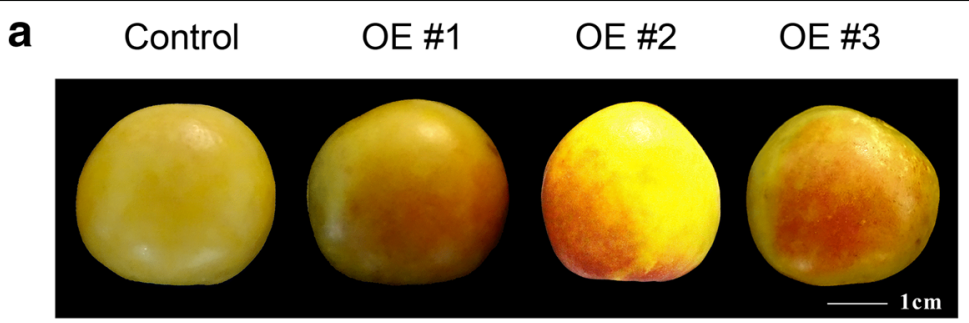

b

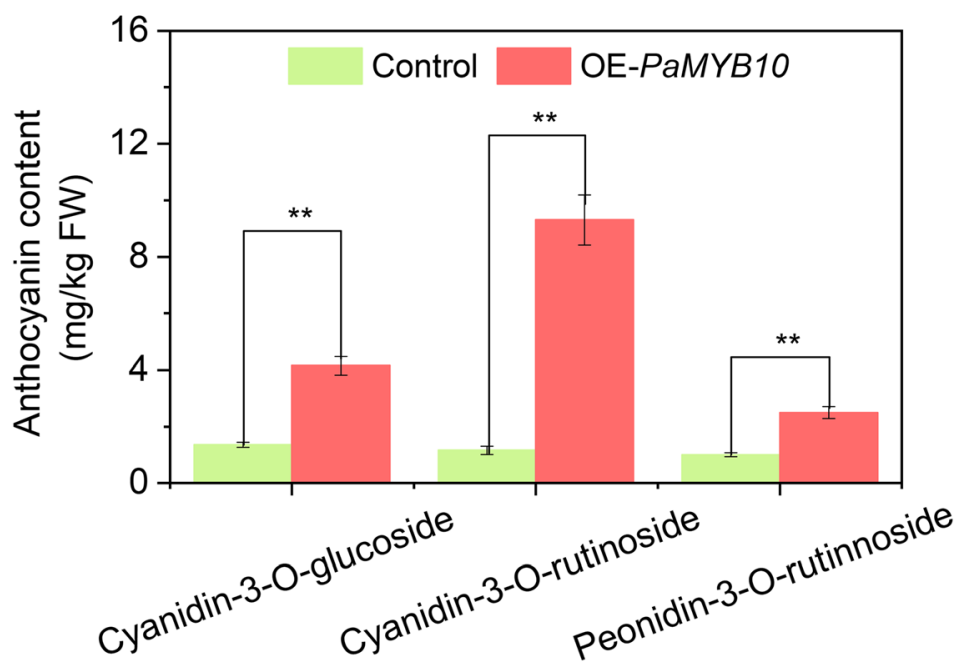

Fig. 9 Transient overexpression in apricot fruit. a Fruits transformed by the empty vector (Control) and overexpression PaMYB10 vector (OE\#1, 2 and 3). $\mathbf{b}$ change in the anthocyanin content in fruit with transient overexpression and the control. Error bars are the \pm SE of the means of three biological replicates. The double asterisk indicates statistically significant differences by Duncan's test at the $1 \%$ level

effluent absorbance was monitored at $525 \mathrm{~nm}$ using the Genesys 10 UV spectrophotometer (Thermo Spectronic, Rochester, NY, USA). The concentration was calculated by the standard curve method and expressed as $\mathrm{mg} / \mathrm{kg}$.FW. Three biological replicates for each sample were used.

\section{RNA extraction and cDNA synthesis}

Total peel RNA was extracted from the fruit using a total RNA kit (RNeasy Plant Mini Kit, Qiagen, Hilden, Germany). RNA samples with an OD260:OD280 between 1.80 and 2.0, an OD260:OD230>1.5 and no discernible degradation were used for cDNA reverse transcription. The RNA was treated with the TURBO DNA-free ${ }^{\mathrm{TM}}$ Kit (Ambion, Austin, TX, USA) following the manufacturer's instructions. Subsequently, firststrand cDNA was synthesized from $1 \mu \mathrm{g}$ of total RNA using the RevertAid First Strand cDNA Synthesis Kit (Invitrogen, Tokyo, Japan) according to the manufacturer's protocol.

\section{Transcriptome sequencing and weighted gene} coexpression network analysis (WGCNA)

'JNL' peel transcriptome sequencing was conducted on the Illumina HiSeq2000 (Illumina, USA) platform.
Sequence assembly and annotation were performed according to our previous work [2]. The WGCNA (v1.29) package in $\mathrm{R}$ was employed to construct coexpression networks and test the association with the anthocyanins. Sequences with an average NRPKM >1 from three replicates were used for the WGCNA. The modules were obtained using the automatic network construction function blockwise with default settings, except that the soft power was 16, the min module size was 30 , and the merge cut height was 0.25 .

\section{Phylogenetic analysis}

A total of 148 MYB sequence fragments were mined from our transcriptome data, and a phylogenetic tree was constructed with MYB transcription factors from other species. After homology alignment, another unrooted phylogenetic tree was constructed using the neighbor-joining (NJ) criteria and verified using the maximum likelihood (ML) method. A total of 1000 bootstrap replicates was performed based on multiple alignments of amino acid (AA) sequence U1373 from JNL and 18 flavonoid-related MYB transcription factors from other higher plants (Additional file 4: Table S3). Multiple protein sequence alignments were performed using ClustalW, and the phylogenetic trees were constructed using MEGA 7.0. 


\section{Quantitative real-time PCR analysis}

Eight pairs of primers (Additional file 5 Table S5) were designed using Primer Premier 5.0, and qRT-PCR was performed using the Light Cycler System (Roche LightCycler ${ }^{\circ}$ 480; Roche Diagnostics). All reactions were performed using the LightCycler 480 SYBR Green I Master Mix (Roche Diagnostics) according to the procedure described by the manufacturer. The qRT-PCR program was $95^{\circ} \mathrm{C}$ for $30 \mathrm{~s}$, followed by 40 cycles at $95^{\circ} \mathrm{C}$ for $5 \mathrm{~s}$ and $60^{\circ} \mathrm{C}$ for $30 \mathrm{~s}$. The reaction volume was $25 \mu \mathrm{L}$ and contained $12.5 \mu \mathrm{L}$ of SYBR Premix Ex Taq (TaKaRa, Dalian, China), $8.5 \mu \mathrm{L}$ of deionized water, $1 \mu \mathrm{L}$ of each primer, and $2 \mu \mathrm{L}$ of diluted cDNA. Three biological replicates for each sample were used. Each sample was normalized using ribosomal RNA and actin as the internal control genes $[39,40]$. The relative expression levels of the eight genes were calculated using the $2^{-\Delta \Delta \mathrm{Ct}}$ method.

\section{Cloning of the full-length PaMYB10 gene}

The PaMYB10 gene was isolated from the cDNA of 'JNL' using a RACE kit (Takara, Dalian, China) (Additional file 6 Table S5). The full-length PaMYB10 was obtained by assembling the 3' RACE and 5' RACE sequences using the BigDye Terminator Version 3.1 Cycle Sequencing Kit (Applied Biosystems) with the ABI PRISM 3100 Genetic Analyzer (Applied Biosystems). The amplification product was cloned into the pMD18$\mathrm{T}$ vector and then transformed into Escherichia coli DH5 $\alpha$ (BioTeke, China). Afterwards, positive colonies were sequenced by Sangon Biological Engineering Company (Shanghai, China).

\section{Subcellular localization}

The PaMYB10 open reading frame (ORF) without the terminator was amplified with special primers (forward: 5'GAGAACACGGGGGACTGGTACCCGGGGATCCA TG GAGGGCTATAACTTGGA-3' and reverse: 5' -ACAGCT CCTCGCCCTTGCTC ACCATGTCGACCCTAGCTTCT TCTGAAACAT-3') and ligated into the pC1301-GFP vector to generate the CAMV 35S:PaMYB10-GFP fusion construct. The recombinant vector (PaMYB10-GFP) and control vector (pC1301-GFP) were both transformed into tobacco by particle bombardment. After 16 to $18 \mathrm{~h}$, fluorescence was detected with a confocal laser scanning microscope (Zeiss LSM 510 META, Jana, Germany).

\section{Overexpression vector construct and transient transformation in apricot fruit}

The overexpression pMDC32 binary vector49 was constructed as previously described [41, 42]. The PaMYB10 coding sequence was PCR amplified from 'JNL' cDNA using the primers 5 '-ATGGAGGGTTATTTCGGTGTGAG-3' (forward) and 5'-TACGTAGGAGATGTTGA
CTAGATCATTGC-3' (reverse) and cloned using the $\mathrm{pCR}^{\mathrm{m}} 8 / \mathrm{GW} / \mathrm{TOPO} \mathrm{TA}$ Cloning Kit (Invitrogen). After sequencing confirmation, the CDS was recombined into the pMDC32 binary vector49 using the Gateway LR Clonase II Enzyme mix (Invitrogen) to produce overexpression vectors. The vectors were transformed into Agrobacterium tumefaciens GV3101 and then resuspended at a final O.D. of 0.8 in liquid MS medium. One milliliter of the suspension was evenly injected into the 'LT' fruit at commercial mature stage. Thirty fruits were injected as the repeat, and fruits were injected with Agrobacterium carrying an empty vector as a control treatment. At 7-12 days after injection, the fruit peel surrounding the injection sites was collected and immediately frozen in liquid nitrogen and stored at $80^{\circ} \mathrm{C}$ for determining the anthocyanin according to the method of above mentioned.

\section{Statistical analyses}

Three biological replicates were performed for all experiments. The least significant difference (LSD) or Duncan's test at the $1 \%$ level was analyzed by DPS (version 2.00; Zhejiang University, Hangzhou, China). Data are expressed as the mean of three biological replicates \pm the standard deviation (SD).

\section{Additional files}

Additional file 1: Table S1. Color indexes of apricot fruits at different developmental stages. (PDF $251 \mathrm{~kb}$ )

Additional file 2: Table S2. Candidate genes involved in anthocyanin biosynthesis in apricot fruit. (PDF $214 \mathrm{~kb}$ )

Additional file 3: Figure S1. Phylogenetic tree of gene sequences for 148 MYB genes from the apricot transcriptome and MYB transcription factors from other species. (PDF $823 \mathrm{~kb}$ )

Additional file 4: Table S3. Flavonoid-related MYB transcription factors using in phylogenetic analysis. (PDF $227 \mathrm{~kb}$ )

Additional file 5: Table S4. The primer sequences for qRT-PCR. (PDF $363 \mathrm{~kb}$ )

Additional file 6: Table S5. The primer sequences for 5' RACE and 3' RACE. (PDF $351 \mathrm{~kb}$ )

\section{Abbreviations}

ANR: Anthocyanidin reductase; BX: Baixing; $\mathrm{CHI}$ : Chalcone isomerase; CHS: Chalcone synthase; DFR: Dihydroflavonol-4-reductase; F3H: Flavanone 3hydroxylase; FLS: Llavonol synthase; HY: Hongyu; JNL: Jianali;

LAR: Leucoanthocyanidin reductase; LDOX: Leucoanthocyanidin dioxygenase; LSD: Least significant difference; LT: Luntaixiaobaixing; PAL: Phenylalanine ammonia-lyase; TF: Transcription factors; UFGT: UDP-flavonoid glucosyl transferase

\section{Acknowledgements}

We thank Julie Caruana (Department of Cell Biology and Molecular Genetics, University of Maryland) for comments on an earlier version of this manuscript.

\section{Authors' contribution}

GHZ conceived the study. WPX designed and performed the experiments. WPX and JF wrote the paper. SKZ and YL analyzed the data. All authors discussed the results and commented on the manuscript. All authors read and approved the final manuscript. 


\section{Funding}

This work was supported by the National Natural Science Foundation of China (31872046). There is on role of the funding bodies in the design of the study and collection, analysis, and interpretation of data and in writing the manuscript.

\section{Availability of data and materials}

The data sets supporting the results of this article are included within the article and its additional files. All sequences obtained from apricots were deposited to the NCBI Sequence Read Archive (SRA) repository under accession number PRJNA506502 and were released on November 27, 2018.

\section{Ethics approval and consent to participate}

Not applicable.

\section{Consent for publication}

Not applicable.

\section{Competing interests}

The authors declare that they have no competing interests.

\section{Author details}

${ }^{1}$ College of Food Science, Southwest University, Chongqing 400715, China. ${ }^{2}$ College of Horticulture and Landscape Architecture, Southwest University, Chongqing 400716, China. ${ }^{3}$ Agriculture National Fruit Tree Germplasm Repository, Xinjiang Academy of Agricultural Sciences, Luntai, Xinjiang 841600 , China.

\section{Received: 25 February 2019 Accepted: 19 June 2019}

\section{Published online: 01 July 2019}

\section{References}

1. Wang Z, Cao JK, Jiang WB. Changes in sugar metabolism caused by exogenous oxalic acid related to chilling tolerance of apricot fruit. Postharvest Biol Technol. 2016;114:10-6.

2. Zhang QY, Feng C, Li WH, Qu ZH, Zeng M, Xi WP. Transcriptional regulatory networks controlling taste and aroma quality of apricot (Prunus armeniaca $\mathrm{L}$. ) fruit during ripening. BMC Genomics. 2019;20:45.

3. Jaeger SR, Harker FR. Consumer evaluation of novel kiwifruit: willingness-topay. Food Agric. 2010;85:2519-26.

4. King MC, Cliff MA. Development of a model for prediction of consumer liking from visual attributes of new and established apple cultivars. J Amer Pomolog Soc. 2002:4:223-9.

5. Palapol Y, Ketsa S, Kui LW, Ferguson IB, Allan AC. A MYB transcription factor regulates anthocyanin biosynthesis in mangosteen (Garcinia mangostana L.) fruit during ripening. Planta. 2009;229:1323-34

6. Harborne JB, Williams CA. Advances in flavonoid research since 1992. Phytochemistry. 2000:55:481-504.

7. Allan AC, Hellens RP, Laing WA. MYB transcription factors that colour our fruit. Trends Plant Sci. 2008;13:99-102.

8. Xu ZS, Huang Y, Wang F, Song X, Wang GL, Xiong AS. Transcript profiling of structural genes involved in cyanidin-based anthocyanin biosynthesis between purple and non-purple carrot (Daucus carota L.) cultivars reveals distinct patterns. BMC Plant Biol. 2014;14:262.

9. Kobayashi S, Goto-Yamamoto N, Hirochika H. Retrotransposon-induced mutations in grape skin color. Science. 2004;304:982.

10. Matsui K, Umemura Y, Ohme-Takagi M. AtMYBL2, a protein with a single MYB domain, acts as a negative regulator of anthocyanin biosynthesis in Arabidopsis. Plant J. 2010:55:954-67.

11. Xu WJ, Dubos C, Lepiniec L. Transcriptional control of flavonoid biosynthesis by MYB-bHLH-WDR complexes. Trends Plant Sci. 2015;20:176-85.

12. Ramsay NA, Glover BJ. MYB-bHLH-WD40 protein complex and the evolution of cellular diversity. Trends Plant Sci. 2005;10:63-70.

13. Koes R, Verweij W, Quattrocchio F. Flavonoids: a colorful model for the regulation and evolution of biochemical pathways. Trends Plant Sci. 2005:10:236-42.

14. Jaakola L. New insights into the regulation of anthocyanin biosynthesis in fruits. Trends Plant Sci. 2013:18:477-83.

15. Schwinn K, Venail J, Shang YJ, Mackay S, Alm V, Butelli E, et al. A small family of MYB-regulatory genes controls floral pigmentation intensity and patterning in the genus Antirrhinum. Plant Cell. 2006;18:831-51.
16. Walker AR, Lee E, Bogs J, Mcdavid DAJ, Thomas MR, Robinson SP. White grapes arose through the mutation of two similar and adjacent regulatory genes. Plant J. 2010;49:772-85.

17. Espley RV, Brendolise C, Chagne D, Kutty-Amma S, Green S, Volz R, et al. Multiple repeats of a promoter segment causes transcription factor autoregulation in red apples. Plant Cell. 2009;21:168-83.

18. Wang ZG, Meng D, Wang AD, Li TL, Jiang SL, Cong PH, et al. The methylation of the PCMYB10 promoter is associated with green-skinned sport in max red Bartlett pear. Plant Physiol. 2013;162:885-96.

19. Uematsu C, Katayama H, Makino I, Inagaki A, Arakawa O, Martin C. Peace, a MYBlike transcription factor, regulates petal pigmentation in flowering peach 'Genpei' bearing variegated and fully pigmented flowers. J Exp Bot. 2014;65:1081-94.

20. Jin HL, Martin C. Multifunctionality and diversity within the plant MYB-gene family. Plant Mol Biol. 1999;41:577-85

21. Zimmermann IM, Heim MA, Weisshaar B, Uhrig JF. Comprehensive identification of Arabidopsis thaliana MYB transcription factors interacting with R/B-like BHLH proteins. Plant J. 2010:40:22-34.

22. Stracke R, Werber M, Weisshaar B. The R2R3-MYB gene family in Arabidopsis thaliana. Curr Opin Plant Biol. 2001:4:447-56.

23. Matus JT, Aquea F, Arce-Johnson P. Analysis of the grape MYB R2R3 subfamily reveals expanded wine quality-related clades and conserved gene structure organization across Vitis and Arabidopsis genomes. BMC Plant Biol. 2008:8:83.

24. Czemmel S, Stracke R, Weisshaar B, Cordon N, Harris NN, Walker AR, et al. The grapevine R2R3-MYB transcription factor VVMYBF1 regulates flavonol synthesis in developing grape berries. Plant Physiol. 2009;151:1513-30.

25. Vimolmangkang S, Han YP, Wei GC, Korban SS. An apple MYB transcription factor, MdMYB3, is involved in regulation of anthocyanin biosynthesis and flower development. BMC Plant Biol. 2013;13:176.

26. Feng SQ, Wang YL, Yang S, Xu YT, Chen XS. Anthocyanin biosynthesis in pears is regulated by a R2R3-MYB transcription factor PyMYB10. Planta. 2010;232:245-55.

27. Kui LW, Bolitho K, Grafton K, Kortstee A, Karunairetnam S, Mcghie TK, et al. An R2R3 MYB transcription factor associated with regulation of the anthocyanin biosynthetic pathway in Rosaceae. BMC Plant Biol. 2010;10:50.

28. Zhai R, Wang ZM, Zhang SW, Meng G, Song LY, Wang ZG, et al. Two MYB transcription factors regulate flavonoid biosynthesis in pear fruit (Pyrus bretschneideri Rehd.). J Exp Bot. 2016;67:1275.

29. Yuan H, Zhang JX, Nageswaran D, Li L. Carotenoid metabolism and regulation in horticultural crops. Hortic Res. 2015;2:15036.

30. Chagne D, Kui LW, Espley RV, Volz RK, How NM, Rouse S, et al. An ancient duplication of apple MYB transcription factors is responsible for novel red fruit-flesh phenotypes. Plant Physiol. 2013;161:225-39.

31. Rahim MA, Busatto N, Trainotti L. Regulation of anthocyanin biosynthesis in peach fruits. Planta. 2014;240:913-29.

32. Gonzalez A, Zhao M, Leavitt JM, Lloyd AM. Regulation of the anthocyanin biosynthetic pathway by the $\Pi \mathrm{G} 1 / \mathrm{bHLH} / \mathrm{Myb}$ transcriptional complex in Arabidopsis seedlings. Plant J. 2010;53:814-27.

33. Broun P. Transcriptional control of flavonoid biosynthesis: a complex network of conserved regulators involved in multiple aspects of differentiation in Arabidopsis. Curr Opin Plant Biol. 2005;8:272-9.

34. Aharoni A, De Vos CHR, Wein M, Sun ZK, Greco R, Kroon A, et al. The strawberry FaMYB1 transcription factor suppresses anthocyanin and flavonol accumulation in transgenic tobacco. Plant J. 2010;28:319-32.

35. Saito K, Yamazaki M. Biochemistry and molecular biology of the late-stage of biosynthesis of anthocyanin: lessons from Perilla frutescens as a model plant. New Phytol. 2002;155:9-23.

36. Tohge T, Nishiyama Y, Hirai MY, Nakajima J, Awazuhara M, Inoue E, et al. Functional genomics by integrated analysis of metabolome and transcriptome of Arabidopsis plants over-expressing an MYB transcription factor. Plant J. 2005:42:218-35.

37. Borevitz JO, Xia YJ, Blount J, Dixon RA, Lamb C. Activation tagging identifies a conserved MYB regulator of phenylpropanoid biosynthesis. Plant Cell. 2000;12:2383-93.

38. Stracke R, Ishihara H, Huep G, Barsch GHA, Mehrtens F, Niehaus K, et al. Differential regulation of closely related R2R3-MYB transcription factors controls flavonol accumulation in different parts of the Arabidopsis thaliana seedling. Plant J. 2010;50:660-77.

39. Marty I, Bureau S, Sarkissian G, Gouble B, Audergon JM, Albagnac G. Ethylene regulation of carotenoid accumulation and carotenogenic gene expression in colour-contrasted apricot varieties (Prunus armeniaca). J Exp Bot. 2005;56:1877-86. 
40. Zhang $X$, Zhang $\sqcup$, Zhang QP, Xu JY, Liu WS, Dong WX. Comparative transcriptome profiling and morphology provide insights into endocarp cleaving of apricot cultivar (Prunus armeniaca L.). BMC Plant Biol. 2017; 17:72.

41. Hawkins C, Caruana J, Schiksnis E, Liu ZC. Genome-scale DNA variant analysis and functional validation of a SNP underlying yellow fruit color in wild strawberry. Sci Rep. 2016;6:29017.

42. Spolaore S, Trainotti L, Casadoro G. A simple protocol for transient gene expression in ripe fleshy fruit mediated by agrobacterium. J Exp Bot. 2001; 52:845-50.

\section{Publisher's Note}

Springer Nature remains neutral with regard to jurisdictional claims in published maps and institutional affiliations.

Ready to submit your research? Choose BMC and benefit from:

- fast, convenient online submission

- thorough peer review by experienced researchers in your field

- rapid publication on acceptance

- support for research data, including large and complex data types

- gold Open Access which fosters wider collaboration and increased citations

- maximum visibility for your research: over $100 \mathrm{M}$ website views per year

At $\mathrm{BMC}$, research is always in progress.

Learn more biomedcentral.com/submissions 